$\mathrm{PM} / 97-44$

GDR-S-003

\title{
Higgs Boson Production in Association with Scalar Top Quarks at Proton Colliders
}

\author{
A. Djouadi, J.-L. Kneur and G. Moultaka \\ Physique Mathématique et Théorique, UMR-CNRS, \\ Université Montpellier II, F-34095 Montpellier Cedex 5, France.
}

\begin{abstract}
We investigate the production of Higgs particles in association with the supersymmetric scalar partners of the top quark at proton colliders. In the minimal supersymmetric extension of the Standard Model, the cross sections for the production of the lightest neutral Higgs boson in association with top squark pairs can be rather large, substantially exceeding the rate for the associated Higgs boson production with top quarks. If the lightest top squark is not much heavier than the top quark, this process will enhance the potential of the CERN Large Hadron Collider to discover the lightest neutral Higgs boson, and will open a window to the study of the Higgs-stop coupling, the potentially largest electroweak coupling in the supersymmetric theory.
\end{abstract}


Supersymmetric theories (SUSY) [1] are the best motivated extensions of the Standard Model (SM) of the electroweak and strong interactions. They provide an elegant way to break the electroweak symmetry and to stabilize the huge hierarchy between the Grand Unification and the Fermi scales. In the Minimal Supersymmetric extension of the Standard Model (MSSM), the $\mathrm{SU}(2) \times \mathrm{U}(1)$ gauge symmetry is broken with two Higgs-doublet fields, leading to the existence of five physical states: two $\mathrm{CP}$-even Higgs bosons $h$ and $H$, a $\mathrm{CP}$-odd Higgs boson $A$ and two charged Higgs particles $H^{ \pm}$[2].

In the theoretically well motivated models, such as Supergravity models [3], the MSSM Higgs sector is in the so called decoupling regime [4 for most of the SUSY parameter space allowed by present data constraints [5]: the heavy $\mathrm{CP}$-even, the $\mathrm{CP}$-odd and the charged Higgs bosons are rather heavy and almost degenerate in mass, while the lightest neutral CP-even Higgs particle reaches its maximal allowed mass value $M_{h} \lesssim 60-130 \mathrm{GeV}$ [6, 7] depending on the SUSY parameters. In this scenario, the $h$ boson has almost the same properties as the SM Higgs boson and would be the sole Higgs particle accessible at the next generation of colliders.

At the CERN Large Hadron Collider (LHC), the most promising channel [8] for detecting such a Higgs particle is the rare decay into two photons, $h \rightarrow \gamma \gamma$, with the Higgs particle dominantly produced via the top quark loop mediated gluon-gluon fusion mechanism $g g \rightarrow h$ [9]. The two LHC collaborations expect to detect the narrow $\gamma \gamma$ peak in the entire intermediate Higgs mass range, $80 \lesssim M_{h} \lesssim 130 \mathrm{GeV}$, with an integrated luminosity $\int \mathcal{L} \sim 100 \mathrm{fb}^{-1}$ corresponding to one year LHC running [8].

Two other channels can be used to detect the Higgs particle in this mass range: the production in association with a $W$ boson, $p p \rightarrow h W$ [10, or in association with top quark pairs, $p p \rightarrow \bar{t} t h$ [11], with the $t$ quarks decaying into $b$ quarks and $W$ bosons [for the latter process, the Higgs boson detection with $h \rightarrow b \bar{b}$ final states looks also promising; see Ref. [12] for instance]. Although the cross sections are smaller compared to the $g g \rightarrow h$ case, the background cross sections are also small if one requires a lepton from the decaying $W$ bosons as an additional tag, leading to a significant signal. Furthermore, the cross section $\sigma(p p \rightarrow \bar{t} t h)$ is directly proportional to the top-Higgs Yukawa coupling, the largest electroweak coupling in the SM. This process would therefore allow the measurement of this parameter, and the experimental test of a fundamental prediction of the SM: the masses of fermions and gauge bosons are generated through the Higgs mechanism.

In this paper, we point out that in supersymmetric theories, which predict the existence of scalar partners to each SM chiral fermion, an additional process might provide a new important source for Higgs particles: the associated production with the scalar partners of the top quark,

$$
p p \rightarrow g g+q \bar{q} \rightarrow \tilde{t} \tilde{t} h
$$

The reason is twofold:

(i) The current eigenstates, $\tilde{t}_{L}$ and $\tilde{t}_{R}$, mix to give the mass eigenstates $\tilde{t}_{1}$ and $\tilde{t}_{2}$ 
which are obtained by diagonalizing the following mass matrix

$$
\mathcal{M}_{\tilde{t}}^{2}=\left(\begin{array}{cc}
m_{\tilde{t}_{L}}^{2}+m_{t}^{2}+D_{L} & m_{t} \tilde{A}_{t} \\
m_{t} \tilde{A}_{t} & m_{\tilde{t}_{R}}^{2}+m_{t}^{2}+D_{R}
\end{array}\right)
$$

where in the off-diagonal entries $\tilde{A}_{t}=A_{t}-\mu / \operatorname{tg} \beta$, with $\operatorname{tg} \beta$ the ratio of the vacuum expectation values of the two-Higgs fields which break the electroweak symmetry, and $A_{t}$ and $\mu$ the soft-SUSY breaking trilinear stop coupling and Higgs mass parameter, respectively. $m_{\tilde{t}_{L}}$ and $m_{\tilde{t}_{R}}$ are the left- and right-handed soft-SUSY breaking top squark masses which, in models with universal scalar masses at the GUT scale, are approximately equal to the common squark mass $m_{\tilde{q}}$; the $D$-terms in units of $M_{Z}^{2} \cos 2 \beta$ are: $D_{L}=$ $\frac{1}{2}-\frac{2}{3} \sin ^{2} \theta_{W}$ and $D_{R}=\frac{2}{3} \sin ^{2} \theta_{W}$. The mixing angle $\theta_{\tilde{t}}$ is proportional to $m_{t} \tilde{A}_{t}$ and can be very large, leading to a scalar top quark $\tilde{t}_{1}$ much lighter than the $t$-quark and all other scalar quarks. The reaction $p p \rightarrow \tilde{t}_{1} \tilde{t}_{1} h$ can be, therefore, more phase-space favored than the corresponding SM-like process $p p \rightarrow \bar{t} t h$.

(ii) Normalized to $2\left(\sqrt{2} G_{F}\right)^{1 / 2}$, the couplings of the top squarks to the lightest Higgs boson read in the decoupling regime,

$$
\begin{aligned}
g_{h \tilde{t}_{1} \tilde{t}_{1}}= & -\frac{1}{2} \cos 2 \beta\left[\cos ^{2} \theta_{\tilde{t}}-\frac{4}{3} \sin ^{2} \theta_{W} \cos 2 \theta_{\tilde{t}}\right] \\
& -\frac{m_{t}^{2}}{M_{Z}^{2}}-\frac{1}{2} \sin 2 \theta_{\tilde{t}} \frac{m_{t} \tilde{A}_{t}}{M_{Z}^{2}} \\
g_{h \tilde{t}_{2} \tilde{t}_{2}}= & -\frac{1}{2} \cos 2 \beta\left[\sin ^{2} \theta_{\tilde{t}}+\frac{4}{3} \sin ^{2} \theta_{W} \cos 2 \theta_{\tilde{t}}\right] \\
& -\frac{m_{t}^{2}}{M_{Z}^{2}}+\frac{1}{2} \sin 2 \theta_{\tilde{t}} \frac{m_{t} \tilde{A}_{t}}{M_{Z}^{2}}
\end{aligned}
$$

involving components which are proportional to $\tilde{A}_{t}$. For large values of the parameter $\tilde{A}_{t}$ [which incidentally make the $\tilde{t}$ mixing angle maximal $\left|\sin 2 \theta_{\tilde{t}}\right| \simeq 1$ ], the latter terms can strongly enhance the Higgs couplings to top squarks, and make it larger than the top quark coupling of the Higgs boson, $g_{h t t} \propto m_{t} / M_{Z}$. Both components would result in an enhancement of the $p p \rightarrow \tilde{t} \tilde{t} h$ rate compared to the $p p \rightarrow \bar{t} t h$ cross section.

In this letter, we analyze the associated production of Higgs bosons with pairs of top squarks at the LHC with a center of mass energy $\sqrt{s}=14 \mathrm{TeV}$. We will concentrate on the case of the lightest $h$ boson of the MSSM in the decoupling regime, and discuss only the production in association with light top squarks. A more detailed discussion will be postponed to Ref. [14].

At lowest order, i.e. at $\mathcal{O}\left(G_{F} \alpha_{s}^{2}\right)$, the process is initiated by the Feynman diagrams shown in Fig. 1. There are 10 diagrams for the $g g$ fusion mechanism [including those with the quartic gluon-squark interaction and the three-gluon vertex] once the various possibilities for emitting the Higgs boson from the squark lines and the crossing of the two 

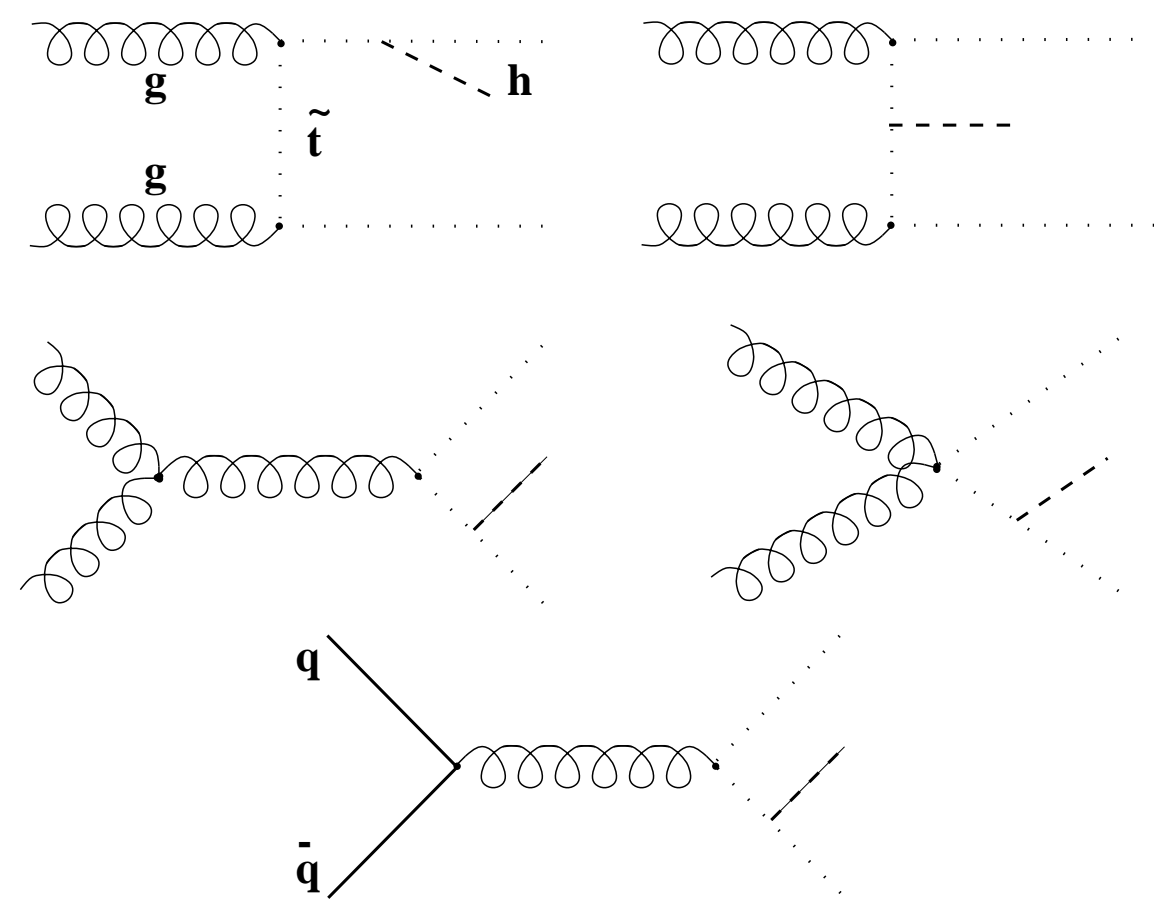

Figure 1: Generic Feynman diagrams for the production of the $h$ boson in association with top squarks via $g g$ fusion and $q \bar{q}$ annihilation.

gluons are added, and 2 diagrams for the $q \bar{q}$ annihilation process. The $\mathcal{O}\left(G_{F}^{3}\right)$ contribution from $\gamma$ and $Z$-boson exchange diagrams, as well as the flavor changing contribution with a gluino exchange diagram are negligible since they are suppressed, respectively, by the additional weak coupling factor and the very small mixing between light quarks and top squarks through the gluino interaction, the mixing being due to weak interactions again. Due to the larger gluon luminosity at high energies, the contribution of the $g g$-fusion diagrams is much larger than the contribution of the $q \bar{q}$ annihilation diagrams at LHC energies, the difference being almost two orders of magnitude for relatively small $\tilde{t}$ mass values. The analytical expressions of the partonic cross section are rather lengthy, and will be given elsewhere [14].

To simplify our numerical analysis, we will assume the left- and right-handed stop mass parameters to be equal, $m_{\tilde{t}_{L}}=m_{\tilde{t}_{R}} \equiv m_{\tilde{q}}$ as is approximately the case in GUT scenarios. For illustration, we have chosen the values $\operatorname{tg} \beta=2$ and 30 . The $h$ boson mass is then calculated as a function of $m_{\tilde{q}}, A_{t}$ and $\mu\left[M_{h}\right.$ is only marginally affected by the variation of the latter parameter] with the pseudoscalar Higgs boson mass fixed to $M_{A}=1$ $\mathrm{TeV}$, and with the full radiative corrections in the improved effective potential approach [7] included. The top quark mass is fixed to $m_{t}=175 \mathrm{GeV}$, and the most recent CTEQ4 parameterizations of the structure functions [15] are chosen.

In Fig. 2, the $p p \rightarrow \tilde{t}_{1} \tilde{t}_{1} h$ cross section [in pb] is displayed as a function of the lightest $\tilde{t}$ mass for the value $\operatorname{tg} \beta=2$, in the case of no-mixing $\tilde{A}_{t}=0\left[A_{t}=200, \mu=400 \mathrm{GeV}\right]$, 
moderate mixing $\left[A_{t}=500\right.$ and $\left.\mu=100 \mathrm{GeV}\right]$ and large mixing $\left[A_{t}=1.5 \mathrm{TeV}\right.$ and $\mu=100 \mathrm{GeV}]$. Note for comparison, that the cross section for the standard-like $p p \rightarrow \bar{t} t h$ process is of the order of $0.6 \mathrm{pb}$ for a Higgs boson mass $M_{h} \simeq 100 \mathrm{GeV}$ [16].

In the case where there is no mixing in the stop sector, $\tilde{t}_{1}$ and $\tilde{t}_{2}$ have almost the same mass [which, up to the small contribution of the D-terms, is constrained to be larger than $\left.m_{t}^{\overline{\mathrm{MS}}}\right]$ and approximately the same couplings to the Higgs boson since the $m_{t}^{2} / M_{Z}^{2}$ components are dominant. The cross section in Fig. 2, which should be then multiplied by a factor of two to take into account the production of both squarks, is comparable to the $p p \rightarrow t \bar{t} h$ cross section in the low mass range $m_{\tilde{t}} \lesssim 200 \mathrm{GeV}$. In scenarios where the $\tilde{t}$ masses are related to the masses of the light quark partners, $m_{\tilde{q}}$, the mass range for which the cross section is rather large is, however, ruled out by present experimental constraints on $m_{\tilde{q}}$ [5].

For intermediate values of $\tilde{A}_{t}$ the two components of the $h \tilde{t}_{1} \tilde{t}_{1}$ coupling interfere destructively and partly cancel each other, resulting in a rather small cross section, unless $m_{\tilde{t}_{1}} \sim \mathcal{O}(100) \mathrm{GeV}$. For some value of $\tilde{A}_{t}$ the $h \tilde{t}_{1} \tilde{t}_{1}$ coupling is zero and the cross section vanishes.

In the large mixing case, $\tilde{A}_{t} \sim 1.5 \mathrm{TeV}, \sigma\left(p p \rightarrow \tilde{t}_{1} \tilde{t}_{1} h\right)$ can be very large. It is above the rate for the standard process $p p \rightarrow \bar{t} t h$ for values of $m_{\tilde{t}_{1}}$ smaller than $220 \mathrm{GeV}$. If $\tilde{t}_{1}$ is lighter than the top quark, the $\tilde{t}_{1} \tilde{t}_{1} h$ cross section significantly exceeds the one for $\bar{t} t h$ final states. For instance, for $m_{\tilde{t}_{1}}=140 \mathrm{GeV}$, corresponding to $M_{h} \sim 76 \mathrm{GeV}, \sigma\left(p p \rightarrow \tilde{t}_{1} \tilde{t}_{1} h\right)$ is an order of magnitude larger than $\sigma(p p \rightarrow t \bar{t} h)$. Note that large values of $\tilde{A}_{t}$, for which in GUT scenarios one needs a sizeable common squark mass parameter $m_{\tilde{q}}$ to avoid color breaking minima, correspond to the "maximal-mixing" scenarios which maximize the $h$ boson mass [7].

In Fig. 3, we fix the lightest top squark mass to $m_{\tilde{t}_{1}}=165 \mathrm{GeV} \sim m_{t}^{\overline{\mathrm{MS}}}$ and display the $p p \rightarrow g g+q \bar{q} \rightarrow \tilde{t}_{1} \tilde{t}_{1} h$ cross section as a function of $\tilde{A}_{t}$ for two values of $\operatorname{tg} \beta=2$ and $\operatorname{tg} \beta=30$. For comparison, the $*$ and $\bullet$ give the standard-like $p p \rightarrow \bar{t} t h$ cross section for $M_{h}=100 \mathrm{GeV}$ and $\operatorname{tg} \beta=2$ and 30 , respectively. For $\operatorname{tg} \beta=30$ the cross section is somewhat smaller than for $\operatorname{tg} \beta=2$, a mere consequence of the increase of the $h$ boson mass with $\operatorname{tg} \beta$ [7]. As can be seen again, the production cross section is substantial for the no-mixing case, rather small for intermediate mixing [becoming negligible for $\tilde{A}_{t}$ values between 200 and $400 \mathrm{GeV}$ ], and then becomes very large, exceeding the reference cross section for values of $\tilde{A}_{t}$ above $\sim 1 \mathrm{TeV}$.

Note that for fixed $\tilde{t}$ mass and coupling, the cross section becomes smaller for larger values of $\tilde{A}_{t}$, if $\tilde{A}_{t} \lesssim \sqrt{6} m_{\tilde{q}}$, because $M_{h}$ increases [7] and the process is less favored by phase-space; in the reverse situation, $\tilde{A}_{t} \gtrsim \sqrt{6} m_{\tilde{q}}$, the $h$ boson mass will start decreasing with increasing $\tilde{A}_{t}$ [reaching values below $M_{h} \lesssim 60 \mathrm{GeV}$ when $\tilde{A}_{t} \sim 1.75$ and $2 \mathrm{TeV}$, for $\operatorname{tg} \beta=2$ and 30 respectively] and the phase-space is more favorable to the reaction.

Let us now discuss the signal for the $p p \rightarrow \tilde{t}_{1} \tilde{t}_{1} h$ process. In most of the parameter 


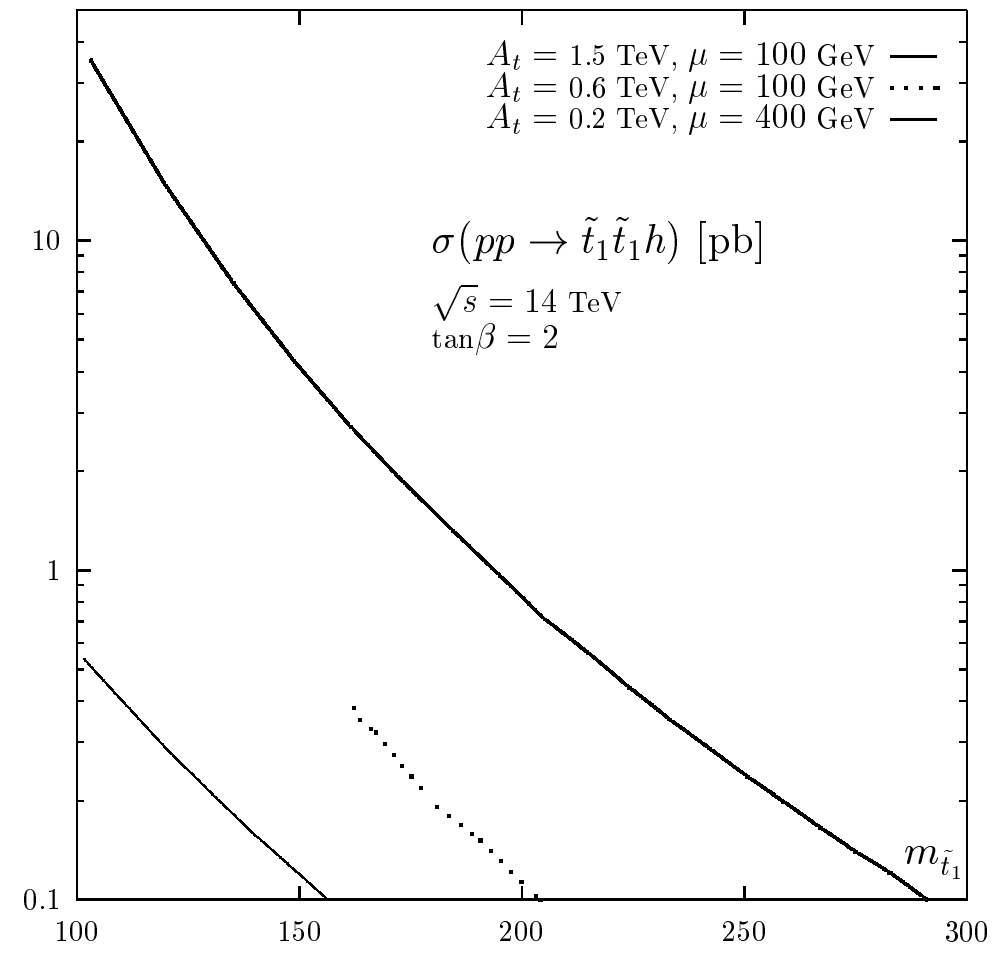

Figure 2: The production cross section $\sigma\left(p p \rightarrow \tilde{t}_{1} \tilde{t}_{1} h\right)$ [in pb] as a function of the $\tilde{t}_{1}$ mass and three sets of $A_{t}$ and $\mu$ values; $\operatorname{tg} \beta$ is fixed to $\operatorname{tg} \beta=2$.

space, the top squark will decay into a $b$ quark and a chargino, $\tilde{t}_{1} \rightarrow b \chi^{+}$, if $m_{\tilde{t}_{1}}<m_{t}+m_{\chi_{1}^{0}}$ where $\chi_{1}^{0}$ is the lightest SUSY particle (LSP), or into a $t$ quark and the LSP, $t \rightarrow t \chi_{1}^{0}$, in the opposite case [17]. In the interesting region where the cross section $\sigma\left(p p \rightarrow \tilde{t}_{1} \tilde{t}_{1} h\right)$ is large, i.e. for relatively light $\tilde{t}_{1}$, the decay mode $\tilde{t}_{1} \rightarrow b \chi^{+}$is dominant, unless the mass difference $m_{\tilde{t}_{1}}-m_{\chi_{1}^{+}}$is very small, in which case the loop induced decay, $\tilde{t}_{1} \rightarrow c \chi_{1}^{0}$, can become competitive. In this region, the strong decay into gluinos does not occur. Assuming that the partners of the leptons are heavier than the lightest chargino, $\chi_{1}^{+}$will mainly decay into the LSP and a real or virtual $W$ boson, leading to the decay

$$
\tilde{t}_{1} \rightarrow b W^{+}+\text {missing energy }
$$

This is the same topology as in the case of the top quark decay, $t \rightarrow b W^{+}$, except that in the case of the top squark there is a large amount of missing energy due to the undetected LSP. If sleptons are also relatively light, charginos decays will also lead to $l \nu \chi_{1}^{0}$ final states. The only difference between the final states generated by the $\tilde{t} \tilde{t} h$ and $t \bar{t} h$ processes, will 


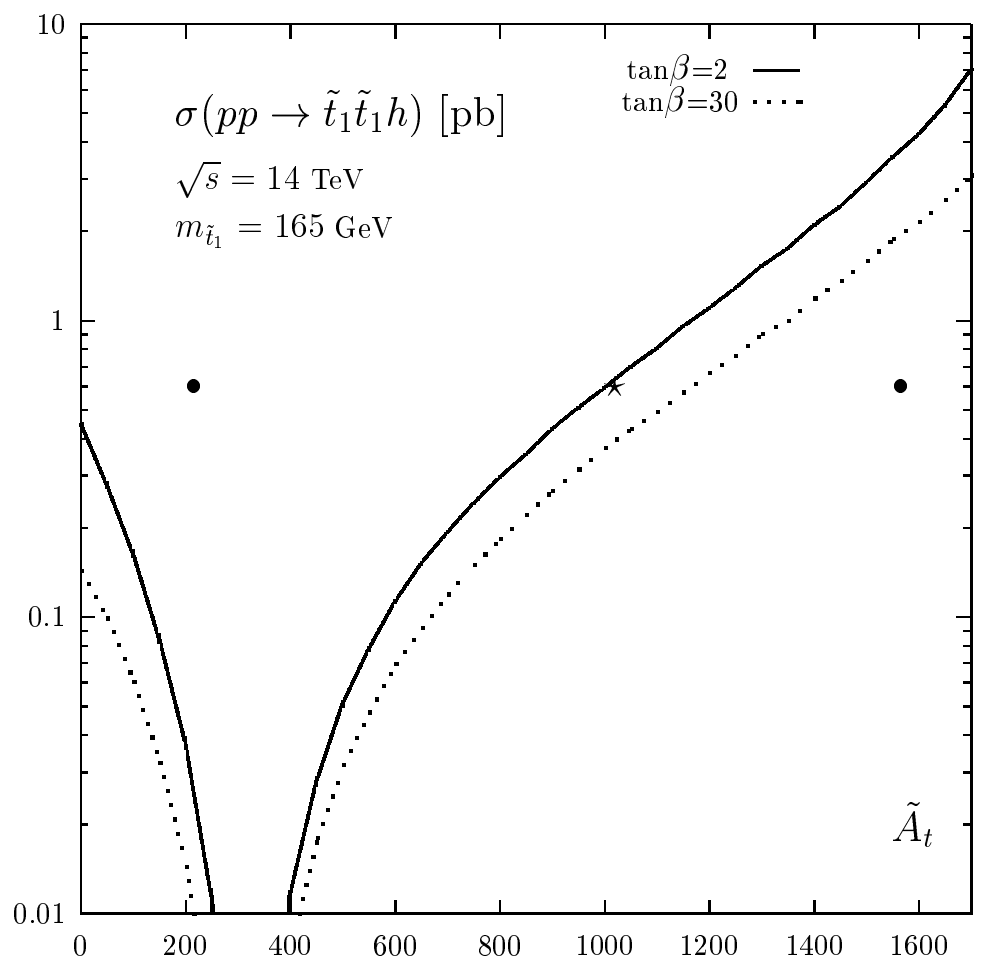

Figure 3: The cross section $\sigma\left(p p \rightarrow \tilde{t}_{1} \tilde{t}_{1} h\right)$ [in pb] as a function of $\tilde{A}_{t}$ for fixed $m_{\tilde{t}_{1}}=165$ $\mathrm{GeV}$ and for $\operatorname{tg} \beta=2,30 . *(\bullet)$ is for $\sigma(p p \rightarrow t \bar{t} h)$ with $M_{h}=100 \mathrm{GeV}$ and $\operatorname{tg} \beta=2(30)$.

be due to the softer energy spectrum of the charged leptons coming from the chargino decay in the former case, because of the energy carried by the invisible LSP.

The Higgs boson can be tagged through its $h \rightarrow \gamma \gamma$ decay mode. In the decoupling limit, and for light top squarks and large $\tilde{A}_{t}$ values, the branching ratio for this mode can be substantially enhanced compared to the SM Higgs boson [18], because of the additional contributions of the $\tilde{t}$-loops which interfere constructively with the dominant $W$-loop contribution. Therefore, $\gamma \gamma+$ charged lepton events can be much more copious than in the SM, and the contributions of the $p p \rightarrow \tilde{t} \tilde{t} h$ process to these events can render the detection of the $h$ boson much easier than with the process $p p \rightarrow t \bar{t} h$ alone.

Although a detailed Monte-Carlo analysis, which is beyond the scope of this letter, will be required to assess the importance of this signal and to optimize the cuts needed not to dilute the contribution of the $\tilde{t} \tilde{t} h$ final states, it is clear that in a substantial area of the MSSM parameter space, the contribution of the top squark to the $\gamma \gamma l^{ \pm}$signal 
can significantly enhance the potential of the LHC to discover the lightest MSSM Higgs boson in this channel. Note that an excess of $\gamma \gamma l^{ \pm}$events would indicate the presence of additional contributions from top squarks to the Higgs boson production. This would be a new and very interesting means to search for top squarks at the LHC, which due to the large QCD background from $\bar{t} t$ production, are otherwise difficult to detect in other channels. Last but not least and as a welcome bonus, an excess of $\gamma \gamma l^{ \pm}$events, compared to the expected rate from the $p p \rightarrow t \bar{t} h$ process, will allow to measure the $h \tilde{t} \tilde{t}$ coupling, the largest electroweak coupling in the MSSM, opening thus a window to probe directly the soft-SUSY breaking scalar potential.

In summary, we have calculated the cross section for the production of the lightest $\mathrm{CP}$-even neutral MSSM Higgs particle in association with the scalar partners of the top quark at proton colliders, $p p \rightarrow g g+q \bar{q} \rightarrow \tilde{t} \tilde{t} h$. The cross section can substantially exceed the rate for the associated production with top quarks, especially for large values of the off-diagonal entry of the $\tilde{t}$ mass matrix which, at the same time, makes $\tilde{t}_{1}$ much lighter than the other squarks and increases its coupling to the $h$ boson. This process can strongly enhance the potential of the LHC to discover the $h$ boson in the $\gamma \gamma+l^{ \pm}$channel, and would open a window for the determination of the important $\tilde{t} \tilde{t} h$ coupling. Finally, this reaction could be a new channel to search for relatively light top squarks at hadron colliders.

\section{Acknowledgments:}

This work is partially supported by the French GDR-Supersymétrie.

\section{References}

[1] For reviews see: H.P. Nilles, Phys. Rep. 110, 1 (1984); H.E. Haber and G.L. Kane, Phys. Rep. 117, 75 (1985).

[2] For a review on the Higgs sector of the MSSM, see J.F. Gunion, H.E. Haber, G.L. Kane and S. Dawson, "The Higgs Hunter's Guide", Addison-Wesley, Reading 1990.

[3] See for instance: J. Amundson et al., Report of the Snowmass Supersymmetry Theory Working Group, hep-ph/9609374; J.F. Gunion, Proceedings of "Perspectives on Higgs Physics, ed. G. Kane, World Sci. Pub., hep-ph/9705282

[4] H.E. Haber, CERN-TH/95-109 and SCIPP-95/15, Proceedings, "Conference on Physics Beyond the Standard Model IV", Lake Tahoe CA 1994.

[5] Particle Data Group, Phys. Rev. D54, 1 (1996); for a recent collection of data, see P. Janot, Int. Euroconference on HEP, Jerusalem 1997.

[6] Y. Okada, M. Yamaguchi and T. Yanagida, Prog. Theor. Phys. 85 (1991) 1; H. Haber and R. Hempfling, Phys. Rev. Lett. 66 (1991) 1815; J. Ellis, G. Ridolfi and 
F. Zwirner, Phys. Lett. 257B (1991) 83; R. Barbieri, F. Caravaglios and M. Frigeni, Phys. Lett. 258B (1991) 167.

[7] M. Carena, M. Quiros and C.E.M. Wagner, Nucl. Phys. B461 (1996) 407; for a summary, see: M. Carena, P.M. Zerwas et al., Higgs Physics at LEPII, CERN yellow report CERN-96-01, hep-ph/9602250.

[8] ATLAS Collaboration, Technical Proposal, Report CERN-LHCC 94-43; CMS Collaboration, Technical Proposal, Report CERN-LHCC 94-38;

[9] H. Georgi et al., Phys. Rev. Lett. 40 (1978) 692; A. Djouadi, M. Spira and P.M. Zerwas, Phys. Lett. B264 (1991) 440; S. Dawson, Nucl. Phys. B359 (1991) 283; M. Spira et al., Nucl. Phys. B453 (1995) 17; S. Dawson, A. Djouadi and M. Spira, Phys. Rev. Lett. 77 (1996) 16.

[10] S.L. Glashow, D.V. Nanopoulos and A. Yildiz, Phys. Rev. D18 (1978) 1724; Z. Kunszt, Z. Trocsanyi and W.J. Stirling, Phys. Lett. B271 (1991) 247.

[11] Z. Kunszt, Nucl. Phys. B247 (1984) 339; J.F. Gunion, Phys. Lett. B253 (1991) 269; W. Marciano and F. Paige, Phys. Rev. Lett. 66 (1991) 2433.

[12] J. Dai, J.F. Gunion and R. Vega, Phys. Rev. Lett 71 (1993) 2699; D. Froidevaux and E. Richter-Was, Z. Phys. C67 (1995) 213.

[13] J. Ellis and S. Rudaz, Phys. Lett. B128 (1983) 248; M. Drees and K. Hikasa, Phys. Lett. B252 (1990) 127.

[14] A. Djouadi, J.L Kneur and G. Moultaka, in preparation.

[15] CTEQ Coll., Phys. Rev. D51 (1995) 4763; Phys. Rev. D55 (1997) 1280.

[16] Z. Kunszt, S. Moretti and W.J. Stirling, Z. Phys. C74 (1997) 479; M. Spira, hep$\mathrm{ph} / 9705337$.

[17] For an update, see e.g. A. Bartl et al., hep-ph/9709252.

[18] A. Djouadi, V. Driesen, W. Hollik and J.I. Illana, hep-ph/9612362 (Z. Phys. C. to appear). 